\title{
Hard-pressed molecular clouds
}

\section{Mark Morris}

JUST about everything - stars, gas, magnetic fields and related activity gets concentrated towards the depths of the central gravitational potential well of our Galaxy. So it is not particularly surprising that the average pressure of the interstellar medium is higher there than in the distant disk of the Galaxy, where we reside. Indeed, observations have long shown that the gas there is substantially warmer and denser than anywhere else in the Galaxy ${ }^{1}$. However, the arguments presented by Spergel and Blitz on page 665 of this issue ${ }^{2}$, that the interstellar pressure within a few hundred parsecs of the nucleus is a full two and a half orders of magnitude larger than that of the solar neighbourhood, threaten to force a reassessment of our notions of the flow of thermal and dynamical energy through the Galactic Centre.

The interstellar medium within a few hundred parsecs of the galactic nucleus (we are 8 kiloparsecs from the centre) is dominated by two markedly contrasting phases (see figure): a planar distribution of molecular clouds with a total mass of about a hundred million solar masses, representing a far greater concentration of cool, dense clouds than is found anywhere else in the Galaxy; and an elliptical cloud of hot, coronal gas which is five or six orders of magnitude less dense than the molecular clouds, but about six orders of magnitude hotter. The coronal phase has been directly observed as an extended source of X-rays, both in the continuum ${ }^{3,4}$, and in the $6.7 \mathrm{keV}$ line of highly-ionized, helium-like iron ${ }^{5}$, which is characteristic of a $10^{8} \mathrm{~K}$ plasma.

The X-ray results had already implied that the coronal phase has a high pressure, but Spergel and Blitz are apparently the first to consider the effect of such pressure on the molecular cloud layer. We have known for about two decades that the widths of molecular spectral lines observed towards Galactic Centre clouds are much larger than in disk clouds $^{1}$. Interpreted in terms of turbulent velocities, as is done by Spergel and Blitz, such linewidths imply a turbulent pressure large enough to be in equilibrium with the high pressure of the surrounding coronal medium. It is also large enough to resist the self-gravity of such clouds and therefore either to impede star formation altogether, or to

allow only low-mass stars to form. This may explain why there is not much evidence of star formation in the Galactic Centre region by comparison with clouds in the galactic disk.

Several important questions are raised by the conclusion that the interstellar pressure is so large in the Galactic Centre. The first is, what is the tremendous source of energy required to maintain the high pressure of the interstellar gas?

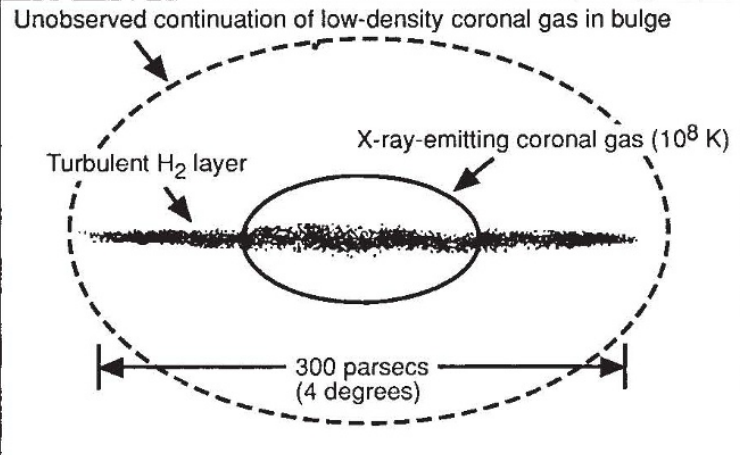

The reservoir of molecular gas (mostly $\mathrm{H}_{2}$ ) in the Galactic Centre is arrayed along the plane of the Galaxy and extends few degrees (150 parsecs) in both directions away from the nucleus. We see it from an almost edge-on perspective (although it may be somewhat tilted), so it appears to the radiotelescopes which are used to map it as a relatively thin line of emitting material. The hot, coronal gas, on the other hand, has been observed with X-ray telescopes to be about half as extended along the galactic plane, but much more broadly distributed into the stellar bulge above the plane. The scale of the central stellar bulge, about 1 kiloparsec, is much larger than either of the observed interstellar constituents, although the coronal gas may extend out into the entire bulge with decreasing (and hence unobservable) density. The density of stars in the central buige (not included in the figure) increases as (radius) ${ }^{-1.8}$ all the way into the inner parsec.

If supernovae are responsible, as many as one supernova every 100 years may be needed in the inner 150 parsecs (ref. 5), comparable with the rate that is often assumed for the entire Galaxy. Alternatively, a single explosion releasing $10^{54}$ ergs of energy within the past $10^{5}$ years could supply this energy ${ }^{5}$, although other evidence for such an event is lacking. Other sources should clearly be considered, such as the gravitational potential energy released by gas accreting into the central region.

Second, what is the scale height of the coronal gas layer? If it is only as large as the observed size of the X-ray emitting regions, then Spergel and Blitz note that the high gas temperature would drive a galactic wind. The mass flux in such a wind would be distressingly large, approaching a few tenths of a solar mass every year. Over the lifetime of the
Galaxy, such a rate of mass loss would imply that the Galactic Centre has lost a quantity of mass comparable to the total mass now present there in the form of both stars and gas. On the other hand, if the scale height were large enough (1 kiloparsec) to permit a hydrostatic description of the coronal gas, then the entire galactic bulge should be luminous in X-rays. Do current observations rule out such a high-luminosity source in the central bulge of the Milky Way or in ther nearby normal galaxies?

Lastly, can the turbulence which presumably supplies the molecular-cloud pressure be maintained? In a dense cloud, turbulence is dissipated on a relatively short timescale, so it must constantly be replenished. What keeps the clouds so effectively stirred up? The interstellar magnetic field is another source of pressure, and perhaps a very important one. At various places within the inner 100 parsecs, the magnetic field strength has been estimated to be of the order of a milligauss ${ }^{6}$, much stronger than is required for pressure equilibrium with the turbulent cloud pressures and the thermal pressure of the coronal phase. Perhaps there is a strong and pervasive magnetic field which dominates the pressure of all phases.

If so, the cloud turbulence might be reinterpreted as magnetohydrodynamic waves there, as it has for clouds in the disk ${ }^{7}$. This might circumvent potential probiems with the short timescales for the dissipation of turbulent ener$\mathrm{gy}$, as the rate of dissipation of the wave energy is probably not as rapid. A strong magnetic field would also confine the coronal gas, and if the field has an organized poloidal geometry, as some evidence may indicate, then it provides a chimney for thermal wind to rise out of, producing, perhaps, the vertical radio structures seen in the nuclear region of a number of spiral galaxies, including our own ${ }^{8}$.

Mark Morris is in the Department of Astronomy, University of California, LoS Angeles, California 90024, USA.

\footnotetext{
1. Güsten, R. in The Center of the Galaxy (ed. Morris, M.) 89-106 (Kluwer, Dordrecht, 1989).

2. Spergel, D. N. \& Blitz, L. Nature 357, 665-667 (1992).

3. Skinner, G. K. et al. Nature 330, 544-547 (1987) 4. Kawai, $N$ et al Astrophys, 330, 130-141 (1988) 5. Yamauchi, S. et al. Astrophys. J. 365, 532-538 (1990) 6. Morris, M. in Galactic and Extragalactic Magnetic Fields (eds Beck, R., Kronberg, P. \& Wielebinski, R.) 361-367 (Kluwer, Dordrecht, 1990).

7. Arons, J. \& Max, C. E. Astrophys. J. 196, L77--L80 (1975)

8. Hummel, E., van Gorkom, J. H. \& Kotanyi, C. G. Astrophys. J. 267, L5-L9 (1983).
}

NATURE · VOL 357 • 25 JUNE 1992 